that certain adipokines have immunomodulatory functions. We therefore analysed localisation of adipokines in tissue, quantified adipokine levels in bronchoalveolar lavage (BAL), and performed in vitro experiments with adipokines.

Methods: Esophagogastroscopy biopsy samples (corpus, oesophagus, antrum) were obtained from SSc patients ( $\mathrm{n}=$ and healthy controls ( $n=$ as well as lung ( $n=e a c h$ ) and skin biopsy samples $(n=e a c h)$. Localisation of adiponectin and visfatin was analysed by immunohistochemistry. Adipokine levels were measured in BAL of healthy controls $(n=$ and patients with SSc ( $n$ "), idiopathic pulmonary fibrosis (IPF) ( $n=$ or EAA ( $n=$ by ELISA. Affymetrix microarray analysis was performed using adiponectin-stimulated SSc skin fibroblasts. Secretion of interleukin 6 (IL-6) by SSc skin fibroblasts, RA synovial fibroblasts (RASF) and osteoarthritis SF (OASF) upon adiponectin and visfatin stimulation was quantified by ELISA.

Results: Expression of adipokines in fibrotic skin, fibrotic lung tissue and the GI tract of SSc patients was increased compared to controls. In the GW of SSc patients, adipokines were localised at non-fibrotic sites of inflammation within the mucosal layer. At the cellular level, adipokines are produced mainly by fibroblasts (vimentin), in the perivascular regions (CD31) and inflammatory cell infiltrates (CD45). Adipokines were strongly expressed in fibrotic lung tissue, and levels in BAL were elevated in SSc. Affymetrix array results showed an increase in expression of chemokines, pro-inflammatory molecules and matrix metalloproteinases, and a decrease in expression of extracellular matrix and adhesion molecules. In SSc skin fibroblasts, secretion of IL- 6 was similarly induced by adiponectin and visfatin as in RASF and OASF.

Conclusions: Organs involved in the pathology of SSc showed increased expression of adipokines. In vitro experiments with SSc skin fibroblasts stimulated with adipokines resulted in increased expression of factors promoting inflammation and breaking down extracellular matrix but decreased expression of fibrogenic factors. These findings suggest a pro-inflammatory but anti-fibrotic effect of adipokines in SSc. Further investigations with focus on the anti-fibrotic potential of adipokines may reveal their involvement in the pathophysiological mechanisms of SSc and lead to new approaches for future therapeutic intervention.

\section{A62 POTENTIAL ADIPOKINE INVOLVEMENT IN SYSTEMIC SCLEROSIS}

Klaus Frommer, Massimiliano Vasile, Tim Schmeiser, Oliver Distler, Steffen Gay, Valeria Riccieri, Andreas Günther, Elke Roeb, Ulf Müller-Ladner, Elena Neumann Internal Medicine and Rheumatology, Justus-Liebig-University of Gießen, Kerckhoff-Klinik, Bad Nauheim, Germany

\subsection{6/ard.2010.129601b}

Background and Objectives Systemic sclerosis (SSc) is a chronic connective tissue disease characterised by excessive fibrosis resulting in its most noticeable symptom, the hardened skin. SSc also affects organs including the gastrointestinal (GI) tract, the vascular system, the lungs and the joints. We could previously show that profibrotic cytokines and infiltration of CD4 $\mathrm{T}$ lymphocytes are increased in the gastric wall (GW) of SSc patients. In rheumatoid arthritis (RA) we found 